\title{
Inhalts - Verzeichnis.
}

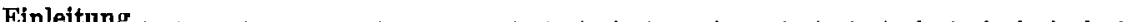

Fufsnoten zur Einleitung . . . . . . . . . . . . . . . . . . . . . . . . . . . . . . . 67

I. ABSCHNITT.

Der Hopfen und die Brauerei in der Geschichte und Sprache . . . . . . . 73

Geschichtliches über den Hopfen und die Bierbrauerei . . . . . . . . . . . . .

1. Ältere Nachriehten aber die Geschichte des Hopfens und des Bieres . . . . . . . . . . 73

2. Eine Erweiterung unseres geschichtlichen Wissens über das Bier und die Verwendung des Hopfens zu seiner Bereitung . . . . . . . . . . . . . . . . . . . . . . . . . 109

Fufsnoten zum I. Abschnitt . . . . . . . . . . . . . . . . . . . . . . . . . . . . . . 138

\section{ABSCHNITT.}

Die botanischen Beziehungen der Hopfenpflanze

Die Arten des Hopfens in der Welt . . . . . . . . . . . . . . . . . . . . . . 144

Die Hopfenpflanze, ihre Organisation und ihre Gebilde . . . . . . . . . . . . . . . . . 152

Der morphologische Aufbau der europäischen Hopfenpflanze (Humulus Lupulus L.) 152

1. Die unterirdische Entwicklung des Hopfens, das Rhizom und die Wurzeln . . . . . . . . 152

2. Die oberirdischen Teile der Hopfenpflanze, Stengel, Äste, Zweige, Blatter, Blütenstände (Hopfendolden oder Zapfen) . . . . . . . . . . . . . . . . . . . . . . . . . . . . 155

Wildhopfen von Untermais bei Meran . . . . . . . . . . . . . . . . . . . . 160

Kulturhopfen aus dem Weihenstephaner Hopfenvarietatengarten . . . . . . . . . . . . . 162

3. Schematische Darstellung von Hopfenästen und Blütenzweigen . . . . . . . . . . . . . . 165

4. Überblick übër die Elemente der Hopfenblütenregion und des Hopfenblütenstandes bei Humulus Lupulus L. . . . . . . . . . . . . . . . . . . . . . . . . . . . . 167

5. Die Laubblätter der Hopfenpflanze . . . . . . . . . . . . . . . . . . . . . . . 170

6. Die Nebenblätter am Stengel der Hopfenptlanze und ihre Anhangsgebilde . . . . . . . . . . 172

Der Bau des Hopfenzapfens oder der Hopfendolde (Trollen, Kätzchen, Köpfe, Zapfen, Kolben, Haupter) . 173

Überblick über die Hopfenzapfen- oder Hopfendolden Typen der Welt . . . . . . . . . . . . . . . 173

Grober Zapfentypus . . . . . . . . . . . . . . . . . . . . . . . . . . . . 174

Mittlerer Zapfentypus . . . . . . . . . . . . . . . . . . . . . . . . . . . . 178

Feinster und feiner Zapfentypus. . . . . . . . . . . . . . . . . . . . . . . . 181

Der Hopfenzapfen ist der Blütenstand der Hopfenpflanze. . . . . . . . . . . . . 187

1. Der Zapfenstiel . . . . . . . . . . . . . . . . . . . . . . . . . . . . . . 187

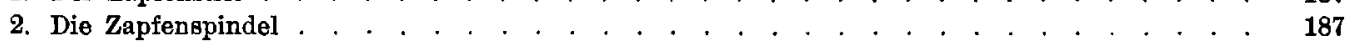

3. Die Ästchen der Spindel oder Blütenstiele . . . . . . . . . . . . . . . . . . . . . . . 189

4. Die Zapfenblatter (Hochblatter), von den Praktikern auch Schuppen genannt . . . . . . . 189 
5. Laubblatter in den sprossenden Hopfenzapfen (Hopfenkonige, Narrenkopfbildung, verlaubte seite Zapfen, Hopfenmandl, Hopfenmänner, Lupel) . . . . . . . . . . . . . . . . . . . 193

6. Die weiblichen Blüten und die Früchte . . . . . . . . . . . . . . . . . . . . . 196

7. Die männliche Hopfenblüte und die Befruchtung . . . . . . . . . . . . . . . . . 203

Fulsnoten zum II Abschnitt . . . . . . . . . . . . . . . . . . . . . . . . . . . . 216

III. ABSCHNITT.

Die Sekretionsorgane des Hopfens als dem Brauer wichtigste Teile der

Hopfenpflanze . . . . . . . . . . . . . . . . . . . . .

Die Sekretionsorgane in den Hopfenzapfen (das Hopfenmehl, der Hopfenstaub, das Lupulin) . . . . . . . . . . . . . . . . . . . . . . . . . . . . . . . . . 219

Die Drüsenvarietäten der grofsen Drüsen und das Mikroskop . . . . . . . . . . . 226

a) Normale, individualisierte Drüsen . . . . . . . . . . . . . . . . . . . 226

a) Individualisierte Drüsen . . . . . . . . . . . . . . . . . . . . . . . . 228

1. Drüsenbecher, gar nicht oder schwach gefüllt . . . . . . . . . . . . . . . 228

2. Randdrüsen, schwach gefullt . . . . . . . . . . . . . . . . . . . . . 228

3. Normale und übervolle Drüsen . . . . . . . . . . . . . . . . . . . . . . . . 280

b) Abnormale, nicht individualisierte Drüsen . . . . . . . . . . . . . . . . 231

4. Halbinnere und innere Drtisen. . . . . . . . . . . . . . . . . . . . . 231

5. Intercellulare Sekretgange oder Milchsaftglinge. . . . . . . . . . . . . . . 234

6. Drüsenzwillinge und Konglomerate . . . . . . . . . . . . . . . . . . . . . 235

7. Uber die Grơfsenverhältnisse der Drüsenvarietäten . . . . . . . . . . . . . 236 Vergleichende Untersuchungen über die Gröfsenverhältnisse der Hopfendrüsen in der Hopfenregion von Spalt in Bayern im Erntejabr 1891 . . . . . . . . . 236

c) Anderweite Drüsen . . . . . . . . . . . . . . . . . . . . . . . . . . 244

8. Drtisen von Humulus cordifolius Miquel . . . . . . . . . . . . . . . . . 244

9. Drüsen von Humulus Japonieus Sieboldt . . . . . . . . . . . . . . . . . . . . . . . . . . . . 245

10. Die Köpfchendrüsen . . . . . . . . . . . . . . . . . . . . . . . . 245

d) Mikrochemische Untersuchungen der Hopfendrüsen und ihres Sekretes . . . 247

e) Reaktion der Salpetersầure ohne Wasser . . . . . . . . . . . . . . . . 249

Fufsnoten zum III Abschnitt . . . . . . . . . . . . . . . . . . . . . . . . . . . . . . 253

\section{ABSCHNITT.}

Die für die Brauerei wirksamen Bestandteile des Hopfens in den Hopfenzapfen und ihre Wirksamkeit bei der Entwicklung und Gestaltung des

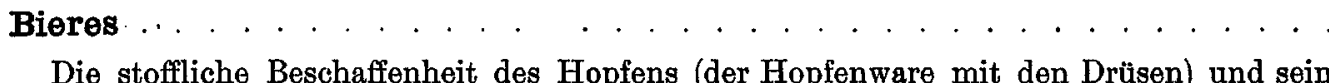
Anteil am Bier

Die Verteilung der Wertbestandteile des Hopfenblütenstandes (der Zapfen oder Dolden) über seine morphologischen Elemente und die Energie ihrer Wirksamkeit . . . . 262

Die Entwicklung der Hopfenkenntnisse für die Zwecke der Brauerei . . . . . . . 275

Die Litteratur über den Hopfen und seine Gebilde . . . . . . . . . . . . . . . 277

Über die Wirksamkeit des Hopfens und namentlich auch seines Sekretes bei der Herstellung des Bieres

Die Zwecke, welche man mit der Zugabe von Hopfen zur Bierbereitung zu erreichen sucht.

1. Beim Brechen der Würze während des Hopfenkochens, um überflüssige Eiweilsstoffe, welche später durch Trübung des Bieres und Minderung seiner Haltbarkeit gefährlich werden können, zum Gerinnen und zur Ausscheidung zu bringen und zu entfernen 
2. Der Hopfen klärt die Würze nicht blofs durch die Ausscheidung der Eiweils. stoffe (Hopfengerbstoffwirkung), sondern auch auf mechanischem Wege, beim lebhaften Kochen durch die Hopfenzapfen in der Pfanne und schliefslich durch das Filtrieren der ausgeschlagenen Würze über den Hopfen. Er unterstützt auch die Sterilisation der Würze

3. Um dem Biere feinen, aromatischen, würzigen Bittergeschmack zu geben

Die Elemente, welche dell Gesamtkomplex des Biergeschmackes bilden . . . . . . . .

Sonstige Umstände, welche den Biergeschmack beherrschen und beeinflussen . . . . .

4. Die Hopfenbittersurrogatfrage in Europa und Nordamerika und die Frage nach Gift im Bier

a) Allgemeines . . . . . . . . . . . . . . . . . . . . . . . . . . . .

b) Vereinigte Staaten von Nordamerika. . . . . . . . . . . . . . . . . . . .

c) England . . . . . . . . . . . . . . . . . . . . . . . . . . . . . . . . . . . . .

d) Europaisches Festland und namentlich Deutschland und Österreich . . . . . . . . . . . . $\quad 320$

Der Alkoholgehalt des Bieres und Bieranalysen . . . . . . . . . . . . . . . 324

Weiteres aber die Hopfensurrogattrage . . . . . . . . . . . . . . . . . . . . . . . . . . 328

5. Will man dem Bier mit dem Hopfen ein feines Aroma (Bouquet, Blume) geben, was durch das ätherische Öl bewirkt wird

. Soll der Hopfen konservierend auf das Bier wirken, indem er die Entwicklung schädlicher Gärungskeime, der Bakterien (Spaltpilze), der Milchsäure-, Buttersäure-, Fäulnis- und Schleim.Gärung hemmt, also eine antiseptische Wirkung ausübt. Auch soll er bei der Nachgärung die normale Gärung durch den Hefepilz verlangsamen. . . . . . . . . . . . . . . . . . . . . . . . . . a) Allgemeines.

Positive und negative Resultate der modernen Kellerbehandlung seit Einfuhrung der Eismaschinen . . . . . . . . . . . . . . . . . . . . . . . . . . .

b) Die Einführung der Eismaschinen als Hauptveranlassung zur Hopfenreduktion

c) Die Weltbierproduktion und der Wandel, den die Hopfengabe aller bierbrauenden Länder unter dem Einflusse der Eiskeller und später der Eis. maschinen und Kaltluftführung, seit 40-50 Jahren durchlaufen hat . . . .

1 Die Weltbierproduktion 1874 "

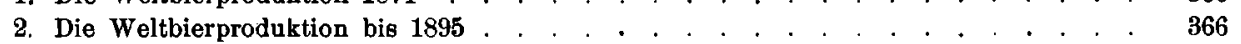

Die Weltbierproduktion bis 1897 . . . . . . . . . . . . . . . . . . . . 367

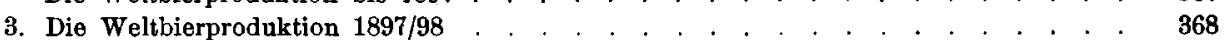

Die Weltbierproduktion $\mathbf{1 8 9 9}$. . . . . . . . . . . . . . . . . . . . . . . . . . . . $\quad . \quad 369$

4. Die Schwankungen der Angaben in der Literatur über den Lander- und Welt-Bedarf an Hopfen in Zentnern à $50 \mathrm{~kg}$. . . . . . . . . . . . . . . . . . . . . . . . . . 369

5. Der Bierverbrauch auf den Kopf der Bevölkerung in Litern. . . . . . . . . . 370

6. Im Anfang und Mitte der 1870 er Jahre bis ca. zum Jahre 1898 ergab die Statistik uber Bierproduktion, Bierkonsumtion, Hopfenverbrauch etc. . . . . . . . . 372

7. Die Weltbierproduktion und die Hopfengabe . . . . . . . . . . . . . . . . . . . . . . . . 374 Die Weltproduktion an Gerste von 1895-1900. . . . . . . . . . . . . . . . 377

8. Europa . . . . . . . . . . . . . . . . . . . . . . . . . . 380

9. Europäischer Kontinent . . . . . . . . . . . . . . . . . . . . . . . $\quad 381$

10. Grolsbritannien . . . . . . . . . . . . . . . . . . . . . . . . . . 381

11. Deutsches Reich . . . . . . . . . . . . . . . . . . . . . . . . . . 393

12. Die Entwicklung der Munchner Brauerei . . . . . . . . . . . . . . . . . 405

Die Entwicklung der Manchner Brauerei von 1810-1897 . . . . . . . . . . . . . 414

Die Entwicklung der Münchner Brauerei seit 1851 . . . . . . . . . . . . . 420

Die Entwicklung der Münchner Grofsbrauerei . . . . . . . . . . . . . . . . . 423

Der Münchner Bierexport . . . . . . . . . . . . . . . . . . . . . . 424

13. Die Entwicklung der Brauerei in Bayern . . . . . . . . . . . . . . . . . 426

Die Bierbrauerei in Bayern seit $1880-1898$. . . . . . . . . . . . . . . . . . . . . . . 439

Die Entwicklung der bayerischen Brauerei seit 1880-1897 . . . . . . . . . . . . . . 441

Bierausfuhr Bayerns nach Mittel- und Nord-Deutsehland . . . . . . . . . . . 444

Brauerei und Hopfenproduktion Bayerns von 1867-1876, . . . . , . . . . . . . 447 
14. Sonstige deutsche Lander . . . . . . . . . . . . . . . . . . . . . . . . . . . 449

a) Norddeutsche Brausteuergemeinschaft . . . . . . . . . . . . . . . . . . . 449

b) Preufsen . . . . . . . . . . . . . . . . . . . . . . . . . . . . . 4454

c) Baden . . . . . . . . . . . . . . . . . . . . . . . . . . . . . . $\quad .455$

d) Elsafs-Lothringen . . . . . . . . . . . . . . . . . . . . . . . . . . 455

e) Württemberg . . . . . . . . . . . . . . . . . . . . . . . . . . . . . . . . . . . . $\quad . \quad 455$

f) Hannover . . . . . . . . . . . . . . . . . . . . . . . . . . . . 456

g) Sachsen . . . . . . . . . . . . . . . . . . . . . . . . . . . . . 456

b) Übrige Zollvereinsstaaten . . . . . . . . . . . . . . . . . . . . . . . . . . . . . . . $\quad .456$

15. Osterreich-Ungarn $\quad . \quad$.

Sonstige europarische Länder . . . . . . . . . . . . . . . . . . . . . . . 465

16. Frankreich . . . . . . . . . . . . . . . . . . . . . . . . . . . . 465

17. Belgien . . . . . . . . . . . . . . . . . . . . . . . . . . . . . . . 466

18. Niederlande (Holland) . . . . . . . . . . . . . . . . . . . . . . . . 468

19. Luxemburg . . . . . . . . . . . . . . . . . . . . . . . . . . . . . . . . . . . 468

20. Die Schweiz . . . . . . . . . . . . . . . . . . . . . . . . . . . . . . . 468

21. Schweden und Norwegen . . . . . . . . . . . . . . . . . . . . . . . 469

22. Dunemark . . . . . . . . . . . . . . . . . . . . . . . . . . . 470

23. Rufsland . . . . . . . . . . . . . . . . . . . . . . . . . . . . . . . . . 470

24. Spanien . . . . . . . . . . . . . . . . . . . . . . . . . . . . . 470

25. Italien . . . . . . . . . . . . . . . . . . . . . . . . . . . . . . . . . . . . . . . . . . . 470

26. Rumbinien . . . . . . . . . . . . . . . . . . . . . . . . . . . . 470

27. Serbien . . . . . . . . . . . . . . . . . . . . . . . . . . . . . 471

28. Bulgarien . . . . . . . . . . . . . . . . . . . . . . . . . . . . 471

29. Griechenland . . . . . . . . . . . . . . . . . . . . . . . . . . . 471

30. Europäische und asiatigche Türkei, Britisch-Indien etc. . . . . . . . . . . . . 471

31. Amerika a) Vereinigte Staaten . . . . . . . . . . . . . . . . . . . . . . 472

32. , b) Brasilien . . . . . . . . . . . . . . . . . . . . . . . . . . 479

33. , c) Mexiko . . . . . . . . . . . . . . . . . . . . . . . . . . . 479

34. Asien . . . . . . . . . . . . . . . . . . . . . . . . . . . . . . 480

Japan . . . . . . . . . . . . . . . . . . . . . 480

d) Die unerwünschten Folgen der modernen Kellerbehandlung des Bieres. Die Unzufriedenheit der deutschen Konsumenten und der Einbruch heller, Pilsener Biere

e) Die wahre Ursache der Notlage in den Hopfenbezirken und die Aussichten für die Zukunft.

Mahnung eines Brauers an die Brauer. . . . . . . . . . . . . . . . . . 495

Der Hopfenbau Mitteleuropas vor und nach $1870 .$. . . . . . . . . . . . . . . 495

f) Die Surrogate, welche in der Brauerei für die konservierende Wirkung der bitteren Hopfenharze angewendet werden

g) Das Hopfenstopfen zur Verstärkung des Aromas und zur Auffrischung der Nachgärung

h) Einfluis des Hopfens auf den Charakter der Haupt- und Nach-Gärung . . .

i) Die physiologische Bedeutung der Hopfenstoffe im Bier auf den menschlichen Organismus

\section{ABSCHNITT.}

\section{Die chemische Natur der Hopfenbestandteile und deren Beziehungen zu} den praktischen Zwecken der Brauerei

Warum die Bestandteile des Hopfens und namentlich des Sekretes einander stofflich so nahestehend sind . . . . . . . . . . . . . . . . . . . . . . . . . . . .

Die wichtigsten chemischen Bestandteile des Hopfens, ihre Eigenschaften und ihr Brauwert 
1. Das ătherische Öl . Seite

a) Nach rein theoretischer Beziehung . . . . . . . . . . . . . . . . . . . . . . . 552

b) Kurze Zusammenfassung der chemischen Studienergebnisse über das ätherische ô . 563

c) Das atherische $\mathrm{Ol}$ in seiner praktischen Bedeutung . . . . . . . . . . . . . . 571

d) Erhaltung des Hopfenöls durch Teilung des Hopfens beim Würzekochen . . . . . . 574

e) Erhaltung des Hopfenöle, des Bouquets, Aromas durch das Darreichen trockenen, frischen, besonders feinen Hopfens in die Lagerfässer . . . . . . . . . . . . 584

2. Der Bitterstoff des Hopfens . . . . . . . . . . . . . . . . . . . . . . 588

a) Nach rein theoretischen Gesichtspunkten . . . . . . . . . . . . . . . . . 588

Die $\beta$-Bittersaure-Krystalle . . . . . . . . . . . . . . . . . . . . . . . . . 602

Die $\alpha$-Bitterskure-Krystalle . . . . . . . . . . . . . . . . . . . . . . . . . 603

b) Kurze Zusammenfassung der chemischen Studienergebnisse über den Hopfenbitterstoff 605

c) Der Hopfenbitterstoff nach praktischen Gesichtspunkten . . . . . . . . . . . 610

3. Die Hopfenharze . . . . . . . . . . . . . . . . . . . . . . . . . . . 635

a) Die allmahliche Entwicklung der verschiedenen Ansichten über die chemische Natur der Harze . . . . . . . . . . . . . . . . . . . . . . . . . . . . .

b) Kurze Zusammenfassung der chemischen Studienergebnisse über die Hopfenharze.

c) Der brautechnische Wert der Bestimmung des Gesamt und Weicbharzgehaltes nebst dem Bittersäuregehalt . . . . . . . . . . . . . . . . . . . . . . . . 651

d) Die Hopfenharze in ihrer praktischen Leistung im Bier . . . . . . . . . . . . 654

e) Das Verhalten der Hopfenharze im alten Hopfen . . . . . . . . . . . . . . . 671

f) Die antiseptische - bakterienfeindliche - Wirkung der Hopfenbittersăure oder des Hopfenharzes . . . . . . . . . . . . . . . . . . . . . . . . . . . 677

1. Die theoretischen Untersuchungen in dieser Frage . . . . . . . . . . . . . . 677

Das Hopfenlupulin als Gift gegen die Virulenz der Sarcina. . . . . . . . . 682

2. Die praktische Seite der antiseptischen Kräfte des Hopfens . . . . . . . 684

3. Kurze Zusammenfassung der Hayduckschen Arbeiten über die Harze des Hopfens und ibre Wirkung auf das Bier. . . . . . . . . . . . . . . 686

4. Die Verwendung der antiseptischen - bakterienfeindlichen - Ejgenschaften des Hopfons bei der Bereitung des Bäckerzouges. . . . . . . . . . . . . . . 686

1. In der Stadt Freising (Oberbayern) . . . . . . . . . . . . . . . . . . . 687

2. In der Stadt Bozen (Südtirol) . . . . . . . . . . . . . . . . . . . . . . 688

3. In anderweiten Gegenden . . . . . . . . . . . . . . . . . . . . . . . . . . . 689

5. Züchtung von Brennereihefe mit Hopfenzusatz. . . . . . . . . . . . . . . 691

6. Die konservierende Wirkung des Säuregehaltes der Hopfensorten und der Einflufs dieses Säuregehaltes auf den Verlauf der Gärung. . . . . . . . . 693

6. Die Gerbstoffe des Hopfens . . . . . . . . . . . . . . . . . . . . . . 698

a) Nach vorwiegend theoretischen Gesichtspunkten . . . . . . . . . . . . . . . . 698

b) Die praktische Brauerei und der Hopfengerbstoff . . . . . . . . . . . . . . . . . . . . . 721

7. Die Hopfenalkaloide. . . . . . . . . . . . . . . . . . . . . . . . . . 732

8. Das Hopfenwachs . . . . . . . . . . . . . . . . . . . . . . . . . . . 736

Sonstige Hopfenbestandteile . . . . . . . . . . . . . . . . . . . . 736

9. Baldriansüure oder Buttersăure (Fettsăure) . . . . . . . . . . . . . . . . . . . . . . 737

10. Esrigghüe . . . . . . . . . . . . . . . . . . . . . . . . . . . . . . . 737

11. Apfelsäure . . . . . . . . . . . . . . . . . . . . . . . . . . . . . . . 737

12. Citronengluure. . . . . . . . . . . . . . . . . . . . . . . . . . 737

13. Cholin . . . . . . . . . . . . . . . . . . . . . . . . . . . . . . 737

14. Eiweifskorper (Stickstoff, Asparagin) . . . . . . . . . . . . . . . . . . . . . 738

15. Trimethylamin . . . . . . . . . . . . . . . . . . . . . . . . . . . . . 739

16. Phloroglucin . . . . . . . . . . . . . . . . . . . . . . . . . . . . . . 740

17. Quercitrin . . . . . . . . . . . . . . . . . . . . . . . . . $\mathbf{7 4 0}$

18. Cyan . . . . . . . . . . . . . . . . . . . . . . . . . . . . . . . . . 740

19. Glykose oder Zucker . . . . . . . . . . . . . . . . . . . . . . . . . . . . . . . . . . . $\mathbf{7 4 0}$

20. Arabinsdure . . . . . . . . . . . . . . . . . . . . . . . . . . . . . . $\mathbf{7 4 0}$

21. Gluten . . . . . . . . . . . . . . . . . . . . . . . . . . . . . . . . 740 
22. Ammoniak . . . . . . . . . . . . . . . . . . . . . . . . . . . . . 740

23. Humusstoffe . . . . . . . . . . . . . . . . . . . . . . . . . . . . . . 740

24. Chloride und Sulfate . . . . . . . . . . . . . . . . . . . . . . . . . . . 740

25. Diastase und Hefe . . . . . . . . . . . . . . . . . . . . . . . . . . . . 740

26. Gummi . . . . . . . . . . . . . . . . . . . . . . . . . . . . . . . . 740

27. Roter Farbstoff . : . . . . . . . . . . . . . . . . . . . . . . . . . . . . 740

28. Fette . . . . . . . . . . . . . . . . . . . . . . . . . . . . . . . . 741

29. Borsänre . . . . . . . . . . . . . . . . . . . . . . . . . . . . . . . 741

Die anorganischen Bestandteile des Hopfens . . . . . . . . . . . . . . . . . . 742

Der Einflufs des Hopfens auf die unorganischen Bestandteile der Würze . . . . . . . . 756

Der Wert des Krautes der Hopfenpflanze bei der Fütterung . . . . . . . . . . . . . 758

Fufsnoten zum V. Abschnitt . . . . . . . . . . . . . . . . . . . . . . . . . . . . . . . . . . . . . 761

VI. ABSCHNITT.

Sonstige Nutzfähigkeit der Hopfenpflanze . . . . . . . . . . . . . . . . . . . . . . 771

Über die physiologisch-medizinische Wirkung des Hopfens . . . . . . . . . . . . 771

Allerlei Verwendungen der Hopfenpflanze und der Hopfenzapfen . . . . . . . . . 778

1. Der Hopfen als Hausmittel . . . . . . . . . . . . . . . . . . . . . . . . 778

2. Der Hopfen als Gewürz . . . . . . . . . . . . . . . . . . . . . . . . . 780

3 Hopfenliköre. . . . . . . . . . . . . . . . . . . . . . . . . . . . . . 781

4. Der Hopfenstengel als Gespinstfaser . . . . . . . . . . . . . . . . . . . . . 782

5. Der Hopfenstengel als Papierstoff . . . . . . . . . . . . . . . . . . . . . . . . . . . . 783

6. Die Hopfenstengel als Garbenbander. . . . . . . . . . . . . . . . . . . . . 784

7. Die jungen Hopfentriebe als Gemüse . . . . . . . . . . . . . . . . . . . . 784

8. Farbstoff im Hopfenstengel . . . . . . . . . . . . . . . . . . . . . . . . . . . 786

9. Hopfenpech . . . . . . . . . . . . . . . . . . . . . . . . . . . . . . 786

10. Der Hopfengeruch als Mittel, Insekten za vertreiben. . . . . . . . . . . . . . . 786

11. Anderweite Verwendungen . . . . . . . . . . . . . . . . . . . . . . . . . . 786

Fưsnoten zum VI. Abschnitt. . . . . . . . . . . . . . . . . . . . . . . . . . . . . . 787

VII. ABSCHNITT.

Die Entwicklung der Bestrebungen, den Hopfen bei der Verwendung in der Brauerei besser auszunutzen, als bisher der Fall war . . . . . . . . 790

Bessere Ausnutzung des Hopfens . . . . . . . . . . . . . . . . . . . . . . . 790

1. Das Prinzipielle in der Sache und allgemeine Ansichten . . . . . . . . . . . . . . 790

2. Zerkleinerung, Zerrei sang, Zerblätterung etc. vor der Verwendung, um dem Lösungsmittel - der Würze - eine gröfsere Oberfluche und dadurch mebr Angriffspunkte darzubieten

3. Hopfenextraktionsmethoden für die augenblickliche Verwendung der Extrakte. . . .

4. Hopfenextrakte als Dauerpriparate . . . . . . . . . . . . . . . . . . . . . . . 814

Untersuchungen über Hopfenextrakte des Handels . . . . . . . . . . . . . . . . . 822

5. Der Zusatz des Hopfens beim Maischen, namentlich bei Verwendung von Rohfrucht . . $\quad 824$

6. Die Anwendung von Hopfenpressen . . . . . . . . . . . . . . . . . . . . . 825

7. Die Hopfentreber als Futtermittel . . . . . . . . . . . . . . . . . . . . . . . 827

8. Die Hopfentreber und die Klarung des Bieres . . . . . . . . . . . . . . . . . 831

9. Hopfentreber als feine Kohle etc. . . . . . . . . . . . . . . . . . . . . . . . . 831

Fufsnoten zum VII. Abschnitt . . . . . . . . . . . . . . . . . . . . . . . . . . . . . 832

VIII. ABSCHNITT.

Die Entwicklung und der gegenwärtige Standpunkt der Bestrebungen, den Hopfen für den Versand und die Aufbewahrung besser zu präparieren, zu verpacken und zu lagern und dadurch seine Verwendungsfähigkeit zu verlängern . . . . . . . . . . . . . . . . . . . . . . . . . . . . . . . . 835

Diè Konservierung des Hopfens . . . . . . . . . . . . . . . . . . . . . . . . 835

1. Allgemeines und die Entwicklung dieser Frage . . . . . . . . . . . . . . . . 835 
3. Die verschiedenen Hopfenverpackungssysteme für Transport, Konservierung und Lagerung .

a) Allgemeines und die Entwicklung der Büchsenverpackung . . . . . . . . . . . . . .

I. Die Verpackung in Quadrat- und Rundballen oder Hopfensucken und deren Lagerung

II. Die Verpackung in Holz- und Metallkisten, wie in freien Quadratballen . . . .

III. Die Packung in Papierfasser oder Rollen (Cylinder) . . . . . . . . . . . . . 877

IV. Die Packung in Holzfässer . . . . . . . . . . . . . . . . . . . . . 878

V. Die Packung in Metallcylinder (Büchsen) . . . . . . . . . . . . . . . . 880

a) Ganze Cylinder and Kisten mit abnehmbarem Boden und Deckel und ungeteiltem Cylindermantel . . . . . . . . . . . . . . . . . . .

b) Metallbuchsen, welche der Quere nach in mehrere Teiletácke zerlegt werden können . . . . . . . . . . . . . . . . . . . . . . . . . . .

c) Metallbúchsen mit einer oder zwei Längsnahten . . . . . . . . . . . . . . . .

4. Das Konservieren des nur gesackten Hopfens durch Aufbewahrung in besonders ausgestatteten Lagerräumen.

5. Untersuchungen über das Verhalten des verschieden behandelten Hopfens beim Lagern . 